Psoriasis

\section{Frühe Therapie bei Kindern und Jugendlichen}

Die Psoriasis ist auch bei Kindern keine Rarität. Der hohe Leidensdruck, der erhebliche Verlust an Lebensqualität und die Perspektive einer lebenslangen einschränkenden Erkrankung mit verpassten Lebens- und Bildungschancen begründen eine dringliche Indikation für eine frühzeitige effektive Therapie.

Psoriasistypisch für Kinder und Jugendliche ist eine Manifestation im Gesicht, am Genital und an den Hand- und Fußflächen. Häufiger als bei Erwachsenen kommt auch eine Psoriasis guttata vor, die bei Kindern oft assoziiert ist mit einer Streptokokkeninfektion. Die Psoriasis-Arthritis tritt bei Kindern anders als bei Erwachsenen typischerweise vor der Hautmanifestation auf, wie Prof. Michael Sticherling, Erlangen, auf einem Satellitensymposium darstellte ${ }^{1}$. Die Psoriasis bedeutet für zwei Drittel der Kinder eine hohe bis sehr hohe Belastung. Auch bei den jugendlichen Patienten ist das Komorbiditätsrisiko erhöht: Diabetes mellitus, Fettstoffwechsel- störungen, Bluthochdruck und Adipositas kommen doppelt so häufig vor wie in der gleichaltrigen Normalbevölkerung, ein Morbus Crohn 3-4-mal häufiger.

Die Psoriasis muss frühzeitig effizient behandelt werden, um die Erkrankung in den Griff zu bekommen und Konsequenzen der Stigmatisierung wie sozialen Rückzug, Schulangst und psychische Belastung zu verhindern. Eine Leitlinie zur Therapie bei Kindern fehlt bisher, auch weil für viele bei der Erwachsenen-Psoriasis eingesetzte Medikamente die Zulassung fehlt. Um die Lücke zu schließen, wurde ein deutscher Experten-Konsensus entwickelt. Die Umsetzung der Empfehlungen in der Praxis ließe jedoch noch sehr zu wünschen übrig, so Prof. Matthias Augustin, Hamburg.

Für Erwachsene mit Psoriasis ist das Spektrum an Therapiemöglichkeiten mit topischen und systemischen Medikamenten sowie Fototherapie so breit wie für keine andere dermatologische Erkrankung.
Doch Pädiater müssen wie so oft weitgehend im Off-Label-Bereich arbeiten. Die Konsensus-Empfehlungen nennen harnstoffhaltige Externa als bevorzugte Basistherapie. Als Standardtherapie werden topische Steroide und Vitamin-D-Derivate empfohlen. Die Indikation zur Fototherapie muss sehr streng gestellt werden. Acitretin kann bei pustulösen Formen eingesetzt werden. Eine systemische Therapie ist indiziert bei einem schweren Krankheitsbild und schlechtem Ansprechen auf die topische Therapie. Methotrexat gilt als Systemtherapeutikum der ersten Wahl. Oft ist auch der Einsatz von Biologika erforderlich. Eine Zulassung unter den Biologika für Kinder hat neben den TNF-alpha-Blockern Etanercept und Adalimumab auch Ustekinumab ${ }^{2}$, und zwar für Kinder ab 12 Jahren.

\section{Dr. Angelika Bischoff, Planegg}

\footnotetext{
${ }^{1}$ Satellitensymposium ,Juvenile Psoriasis - Welche Chancen bieten die neuen Therapieoptionen?" im Rahmen der DGKJ-Jahrestagung, September 2015, München, Veranstalter: Janssen-Cilag $\mathrm{GmbH}$

${ }^{2}$ Stelara ${ }^{\circledR}$, Janssen-Cilag GmbH, Neuss
}

ner unter Monotherapie). Gegenüber der Imilimumab-Monotherapie reduzierte diese Kombinationstherapie das Progressions- bzw. Mortalitätsrisiko um 60\%. Bei einer Follow-up-Dauer von mindestens 11 Monaten kam es nicht zur Progression. Das Sicherheitsprofil der Kombinationstherapie entsprach dem bereits veröffentlichter Studien: Unter den Nebenwirkungen Grad 3/4 wurden am häufigsten Colitis (17\%), Diarrhö (11\%) und ein Anstieg der Alanin-Aminotransferase (11\%) beobachtet.

\section{Ute Ayazpoor, Mainz} berichtete Prof Stephan Grabbe, Mainz, auf einem Satellitensymposium ${ }^{1}$.

Die Kombination des PD1-Checkpoint-Inhibitors Nivolumab mit dem gegen CTLA4 gerichteten Ipilimumab ${ }^{2}$ führte bei Patienten mit therapienaivem, fortgeschrittenem Melanom und BRAF-Wildtyp zu einer Gesamtansprechrate von 61 vs. $11 \%$ unter einer Monotherapie mit Ipilimumab, stellte Grabbe aktuelle Studienergebnisse vor $^{3}$. Immerhin $22 \%$ der mit der Kombinationstherapie behandelten Patienten mit BRAF-Wildtyp erzielten ein vollständiges Ansprechen (dagegen kei-

${ }^{1}$ Satellitensymposium „Immuno-oncology: A New Pillar in Cancer Therapy Emerging“ im Rahmen des 13. Jahrestreffens der Association for Cancer Immunotherapy (CIMT), Mai 2015, Mainz, Veranstalter: Bristol-Myers Squibb GmbH \& Co. KGaA

${ }^{2}$ Yervoy $^{\circledR}$, Bristol-Myers Squibb GmbH \& Co. KGaA, München
${ }^{3}$ Postow MA et al. N Engl J Med 2015; 372: 2006-2017 bensverlängerung bei fortgeschrittenen Melanom ermöglicht, wurde die Immuncheckpoint-Blockade entwickelt. Inzwischen werden mehrere Substanzen geprüft bzw. stehen zur Verfügung, die auf verschiedene Checkpoint-Signalwege ab- 